\title{
Internal Proper Motion of 6.7 GHz Methanol Masers in Ultra Compact HII Region S269
}

\author{
S. Sawada-Satoh ${ }^{1}$, K. Fujisawa ${ }^{2}$, K. Sugiyama ${ }^{2}$, \\ K. Wajima ${ }^{2}$ and M. Honma ${ }^{3}$ \\ ${ }^{1}$ Mizusawa VLBI Observatory, NAOJ, Mizusawa, Oshu, Iwate, 023-0861 Japan \\ email: satoko.ss@nao.ac.jp \\ ${ }^{2}$ Dept. of Physics, Yamaguchi University, Yoshida, Yamaguchi, 753-8512 Japan \\ ${ }^{3}$ VERA Project, NAOJ, Osawa, Mitaka, Tokyo, 181-8588 Japan
}

\begin{abstract}
We present the internal proper motion of 6.7-GHz methanol masers in S269, an Ultra Compact HII region. The maser distribution in S269 consists of several maser groups, and the spatial structure of the main groups A and B are consistent with the past VLBI image. The remarkable result of comparing the two VLBI maps is that $6.7-\mathrm{GHz}$ methanol maser distribution and velocity range within each group have been kept for eight years. Angular separation between the two groups A and B increases by 3.6 mas, which corresponds to a velocity of $11.5 \mathrm{~km} \mathrm{~s}^{-1}$.
\end{abstract}

Keywords. masers - ISM: HII regions - stars: formation

\section{Introduction}

S269 is an Ultra Compact HII region in the outer Galaxy (Sharpless 1959), and harbors two bright near-IR sources, IRS1 and IRS2. In IRS2, several star-forming activities such as $\mathrm{OH}, \mathrm{H}_{2} \mathrm{O}$ masers and $\mathrm{CO}$ wings have been detected. Recent near-IR images imply that several $\mathrm{H}_{2}$ knots are distributed across IRS2, which suggest a bipolar outflow, powered by sources in IRS2 (Jiang et al. 2003). The past VLBI observations of the 6.7-GHz methanol maser in S269 detected two groups (A \& B), separated by 60 mas in 1998 November (Minier et al. 2000). The observations have suggested that the $6.7-\mathrm{GHz}$ methanol masers are probably associated with IRS2. We observed the 6.7-GHz methanol masers in S269 in 2006 September using the Yamaguchi 32-m telescope and the Japanese VLBI Network (JVN).

\section{Maser distributions and internal proper motion}

The spectrum detected with the Yamaguchi 32-m telescope reveals the brightest peak at velocity of $15.2 \mathrm{~km} \mathrm{~s}^{-1}$, and blue-shifted and red-shifted spectral components at peak velocities of 14.7 and $15.9 \mathrm{~km} \mathrm{~s}^{-1}$, which are consistent with the past single-dish observations (Szymczak et al. 2000, Goedhart et al. 2004). Flux densities at the peaks of 14.7, 15.2 and $15.9 \mathrm{~km} \mathrm{~s}^{-1}$ are 9.8, 19.0 and $6.1 \mathrm{Jy}$, respectively. The flux density values agree well with an extrapolation of the sinusoidal time variation of the methanol maser emission (Goedhart et al. 2004).

The maser distribution consists of several maser groups. The most luminous maser spot at $15.2 \mathrm{~km} \mathrm{~s}^{-1}$ belongs to group B. The velocity range of group B is 15.02 to $15.38 \mathrm{~km} \mathrm{~s}^{-1}$. Group $\mathrm{A}$ is the second brightest group, and consists of two maser spots at velocities of 14.67 and $14.85 \mathrm{~km} \mathrm{~s}^{-1}$, and is located $\sim 60$ mas east from group B. The red-shifted maser component is marginally detected and divided into two spectral channels at velocities of 15.73 and $15.90 \mathrm{~km} \mathrm{~s}^{-1}$ with the JVN. The spots form another 

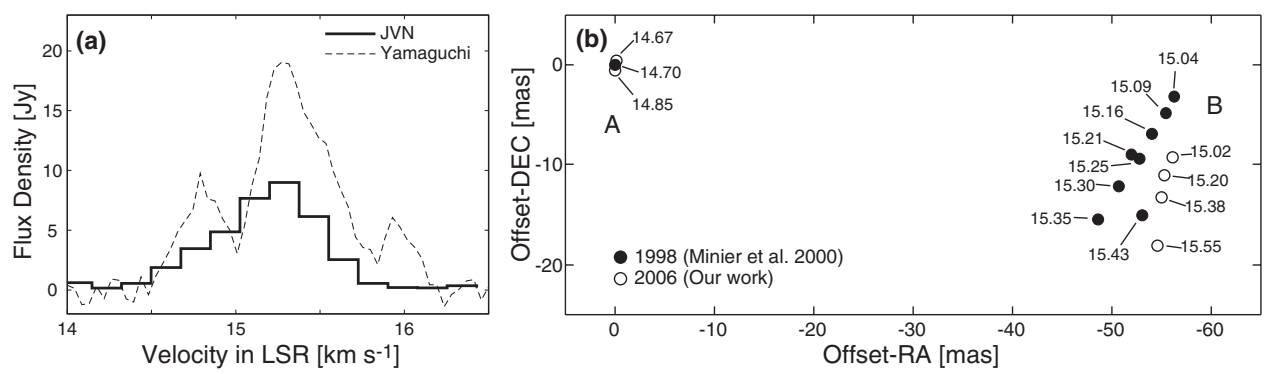

Figure 1. (a) The cross-power spectrum obtained from our JVN observations on 2006 September 10 (Solid line), and the spectral profile measured with the Yamaguchi 32-m telescope averaged spectra observed from 2006 September 4 to 7 (Dashed line). Velocity resolution is $0.178 \mathrm{~km} \mathrm{~s}^{-1}$ and $0.044 \mathrm{~km} \mathrm{~s}^{-1}$, respectively. (b) Superposed maps of $6.7-\mathrm{GHz}$ methanol maser distribution in 1998 (Minier et al. 2000) and 2006 (our work). For the distribution in 1998, Coordinate origin $(0,0)$ is set to (i) the maser spot at velocity of $14.70 \mathrm{~km} \mathrm{~s}^{-1}$ in 1998 , and (ii) the middle point of the two spots at velocity of 14.67 and $14.85 \mathrm{~km} \mathrm{~s}^{-1}$ in 2006 .

group, -95 mas west from group A. On the other hand, the blue-shifted maser spots at velocities of $14.32,14.50,14.67$ and $14.87 \mathrm{~km} \mathrm{~s}^{-1}$ have a wider distribution, spanning 250 mas along the southeast-northwest direction. Those maser spots except for groups A and B are not visible in the EVN observations of 1998 November (Minier et al. 2000). The maser peaks at 14.7 and $15.9 \mathrm{~km} \mathrm{~s}^{-1}$ could be at the minimum phase in intensity at that time, as Goedhart et al. (2004) has shown.

The structures of groups A and B are consistent with the past VLBI image of Minier et al. (2000). The remarkable result of comparing the two VLBI maps is that the $6.7-\mathrm{GHz}$ methanol maser distribution and velocity range within each group have been maintained for eight years. Superposition of the two VLBI maps (Figure 1b) reveals that the distance between the two groups A and B increased by 3.6 mas over 7.8 years, which corresponds to a velocity of $11.5 \mathrm{~km} \mathrm{~s}^{-1}$. The motion is along the direction of position angle $\sim 80^{\circ}$, almost parallel to the east-west direction.

The velocity gradient in the overall distribution could roughly be seen along the direction of position angle of $\sim 80^{\circ}$. However, a more rigorous inspection for the individual maser groups indicates that the velocity gradient is not simple. Velocities of the individual groups spread across the direction at a position angle of $\sim 80^{\circ}$.

The increasing angular separation between groups A and B could trace the outflow powered by IRS2. In order to confirm the movement, we have performed a follow up observation of the 6.7-GHz methanol masers in October 2011.

\section{Acknowledgment}

We are grateful to the JVN team for their support during the observations.

\section{References}

Goedhart, S., Gaylard, M. J., \& van der Walt, D. J. 2004, MNRAS, 355, 553

Jiang, Z., Yao, Y., Yang, J., Baba, D., Kato, D., Kurita, M., Nagashima, C., Nagata, T., Nagayama, T., Nakajima, Y., Ishii, M., Tamura, M., \& Sugitani, K. 2003, ApJ, 596, 1064 Minier, V., Booth, R. S., \& Conway, J. E. 2000, A\&A, 362, 1093

Sharpless, S. 1959, ApJS, 4, 257

Szymczak, M., Hrynek, G., \& Kus, A. J. 2000, A\&A, 143, 269 